\title{
A Suggestion to Improve Timely Feedback of Infectious Disease Surveillance Data at a Provincial Level in South Korea
}

\author{
Seon-ju Yi*, Gichan Park, Hwami Kim, SoYoon Min, Jee soo Suh, Soojung Jo, Daeun \\ Jeong, Young-Man Kim and Heeyoung Lee
}

Surveillance Team, Gyeonggi Infectious Disease Control Center, Seongnam-si, Korea (the Republic of)

\section{Objective}

This study will explore the timeliness of the Korean National Notifiable Disease Surveillance System (KNNDSS) at provincial Level, and suggest a reasonable duration for publication the weekly reports to improve timely feedback of infectious disease surveillance data to physicians and community.

\section{Introduction}

In South Korea, the NNDSS is organized at three levels: local, provincial, and central. At the local level, physicians report the cased to the Public Health Center (PHC) and PHC conduct control measures. At the provincial level, the PHC reports the cases to the Department of Health (DOH) of the province and DOH obliged to report the cases to the Korea Centers for Disease Control and Prevention (KCDC) and feedback of the surveillance data to PHC and physicians. At the central level, the Disease Web Statistics System (http://is.cdc.go.kr/ dstat/index.jsp) provides real-time data on 54 national notifiable infectious diseases [1].

Although there are variations according to the disease and surveillance step, the KNNDSS generally functions well in terms of timeliness and $\mathrm{Yu}$ et al. reported that $89.7 \%$ of mumps, one of the most incident contagious disease in South Korea, reported in 15 days after the physician's notification [2]. To improve the timeliness of feedback at the provincial level, we explored the KNNDSS data and suggested an evidence based duration for publication of the weekly reports, in consideration of reducing the publication schedule.

\section{Methods}

We analyzed the reported infectious disease surveillance data( $(\mathrm{N}=23,486)$ at Gyeonggi province in 2014. Time points recorded in the KNNDSS data include dates of onset, diagnosis, doctor's notification to the $\mathrm{PHC}$, $\mathrm{PHC}$ reporting to the $\mathrm{DOH}$, and $\mathrm{DOH}$ reporting to the KCDC [2]. Using these dates, we defined the time lags in days from physician's notification to central appraisal, and the number of cases reported last week was summarized by the days of notification and publication schedule.

\section{Results}

The average time lags were 9.6 days $(\mathrm{SD}=30.3)$ from physician's notification to central appraisal.

$21,108(92.73 \%)$ of reported cases were finished central appraisal till next Friday. 96.82\%, 92,17\% and $90.86 \%$ of cases notified on Sunday, Wednesday and Saturday, respectively, could be covered on next Friday.

$20,491(90.02 \%)$ of reported cases were finished central appraisal till next Wednesday. $96.13 \%, 90.47 \%$ and $83.60 \%$ of cases notified on Sunday, Wednesday and Saturday, respectively, could be covered on next Wednesday.

Otherwise, only $15,684(68.90 \%)$ of reported cases were finished central appraisal till next Monday. $95.16 \%, 80.91 \%$ and $4.54 \%$ of cases notified on Sunday, Wednesday and Saturday, respectively, could be covered on next Monday.

\section{Conclusions}

Our study suggested the utility of the assessment of time-lag distributions for the feedback strategies to improve surveillance and regional infectious disease controls.
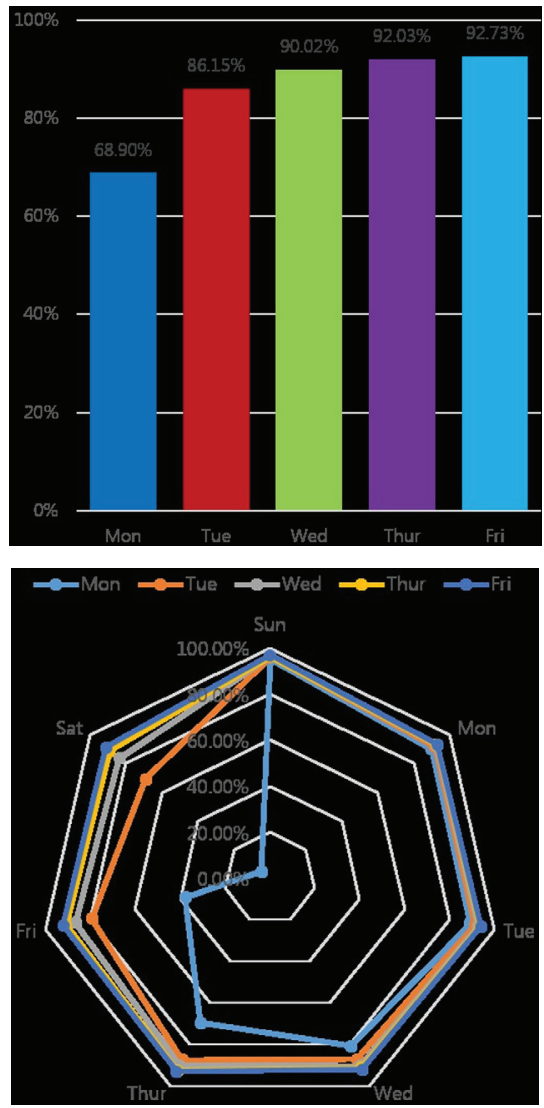

\section{Keywords}

Korean National Notifiable Disease Surveillance System; Notifiable Disease; Timeliness; Weekly report

\section{References}

[1] Park, Sunhee, and Eunhee Cho. "National Infectious Diseases Surveillance data of South Korea." Epidemiology and health 36 (2014).

[2] Yoo, Hyo-Soon, et al. "Timeliness of national notifiable diseases surveillance system in Korea: a cross-sectional study." BMC Public Health 9.1 (2009): 93.

\section{*Seon-ju Yi}

E-mail: yiseonju@gidcc.or.kr 